\title{
Analysis on Selection Orientation and Degradation of Automobile Engine Lubricant
}

\author{
Liang Jiao \\ Baoji Vocational Technology College, Baoji, 721001, China
}

\begin{abstract}
In frequent application, automobile engine lubricant will gradually degrade. Upon careful selection, lubricant under proper specification will improve the original running performance of car body and lengthen the service life of accessories in the automobile structure. Hidden degradation inclination includes fundamental reasons at various levels. It is necessary to specify original factors for degradation of lubricant. For selection in the initial stage, characters of lubricant, inherent physicochemical indexes of automobile and rules for replacement of lubricant should be taken into account. The optimal oil draining period should be preset, so as to guarantee the added lubricant and maintain the optimal performance.
\end{abstract}

Key words: automobile engine lubricant, selection orientation, degradation

The optimal lubricating property is the original guarantee of usual running of engine. Upon application for a long period, however, lubricant will be inclined to degradation under the state of high-temperature oxidation, and its original physicochemical property will be changed. Selection of lubricant is closely correlated with potential degradation state. Whether the present replacement period is of proper character is directly associated with the service life and durability of engine. Therefore, we should identify degradation factors, take multiple measures for the feature of application and prevent excessively fast degradation.

\section{Overall lubricating needs}

The complex situation is highlighted in daily running process of car body. For lubricant matched with engine, the proposed conditions are relatively rigorous, and the range of temperature replacement is relatively large. For instance, in terms of car body in the open air, the engine oil temperature will be approximate to the air temperature in the cold period. Upon long-distance running or addition of weigh load, the air cylinder connected with the piston will accumulate relatively high engine oil temperature. Under the circumstance of low temperature, emphasis should be laid to inherent temperature feature of lubricant for the advantage of 
usual car body startup. Only in this way, engine after heating can maintain conventional lubrication.

Inherent structure of engine will highlight difference. Moreover, difference will be also hidden in proposed test conditions. Investigation should be made on engine oil startup \& pumping feature under low temperature and lubrication feature under high temperature. Moreover, automobile brands in different features should also follow practice and be provided with proper lubricant.

\section{State peculiar to friction accessories}

Friction modules installed on engine can be divided into lubricating states in different features. Subdivided categories of modules include cam, tappet connected with the air valve, air cylinder set matched with the piston and connecting rod connected with the crankshaft. During marching forward of car body, shaft neck and installed bearing are under the usual lubricating state. In this stage, the friction coefficient is closely associated with the engine oil viscosity. If lubricating conditions within the boundary range are neglected, the oil body with the feature of low viscosity will shrink friction loss in ordinary days. Camshaft and installed valve lifter will also follow preset indexes. Lubricant will be properly added. Lubrication condition set at the boundary and dynamic pressure lubrication in the elastic fluid structure should be taken into overall account.

It is very difficult to add lubricant into the air cylinder in piston and system structure. The friction surface installed in engine is approximate to conventional bearing. However, it is very difficult for the revolving direction replacement under the state of high temperature to maintain fluid lubrication under the optimal situation. Inherent outer wall of the air cylinder will often highlight bruise. If the preset viscosity is relatively low, abrasion will be worsening. Piston ring and inherent outer wall of the air cylinder will highlight the resistance of start under high temperature. The hot break-out torque under this range and inherent viscosity of oil body highlight the inverse ratio association. Thus, it can be seen that the lubrication viscosity related to friction modules is of real requirements at different levels. To save consumed fuels, we should select lubricant with the optimal viscosity upon practical contrast according to preset internal framework and running conditions of engine.

\section{Notes within the range of selection}

(I) Select proper mark 
The emphasis within the selection range is laid on the selection of viscosity mark under proper specification. Viewed from the current situation, the yield of multi-level oil is relatively small. In the relatively cold range, the optimal mark should be selected according to changing temperature, if the temperature of car body with the feature of cargo carrying fails to meet the low temperature state within this range. The reason is that it is very difficult for inherent temperature of non-compound oil to correspond to high and low temperature in seasonal change. Factors to be considered in identification and selection of viscosity mark include temperature difference in the seasonal period, normal running of car body and technical indexes of engine.

The formation of oil film with the lubrication feature is closely correlated with fundamental viscosity. Under this state, viscosity indexes under proper framework should be selected. The optimal indexes should guarantee that oil body also has enough thickness of oil film and high-level thickness of oil film under the preset high temperature. In usual running of engine, friction accessories with combined feature are of lubrication action and corresponding sealing feature. Under relatively low temperature of oil body, the starting performance under low temperature should be preset. The optimal viscosity selected is usually correlated with ambient temperature in the range, load of engine accessories and revolving speed at various stages.

For oil body mark under the feature of high viscosity under low temperature, preset starting characteristic and pumping feature are of certain deviation. After real starting, the rate is relatively low, highlighting abrasion of accessories and adding fuel internal friction within the rated range. On the contrary, the lubricant with low viscosity can shrink friction power within the system. At the same time, the retention feature of oil body is the optimal, and thermal energy consumed by friction is less. The flow rate peculiar to lubricant body is increased, highlighting the cooling value at the optimal level.

For this oil body with viscosity property, it suspends and carries multiple sundries which will be filtrated by a filter. During usual activation, oxidative deterioration within this range can be reduced. For the piston ring installed in engine, the sealing level should be preset. Under usual abrasion state, selected lubricant should be of the lowest viscosity.

(II) Select quality grade 
Quality grade unique to lubricant indicates the use performance. The grade should be followed to identify lubricant. The basis for determination of oil body grade includes common operating condition of engine, multiple devices attached to exhaust and preparation period of engine. Usually, proposed high level can replace quality grade in a relatively low range. However, present low level can not be used to replace the original high level. If replaced like this, damage will be caused to engine accessories.

To reduce pollution, laws and regulations within the exhaust pipe control field are gradually improved. To adapt to this state, certain devices within the additional framework should be added into the air inlet part of engine accessories for the convenience of reduction to emission of poisonous substances. Added accessories degrade the original working conditions and preset running standards under a higher level. For usual running, additive mixed by certain formula should be added into purchased oil body to form conformed oil products. Only in this way, lubricating requirements attached to accessories can be satisfied. Additional devices installed in the inlet and outlet system are closely associated with lubrication property.

For engine without additional accessories, the quality of oil body selected includes lubricant at the level of QB and lubricant under multi-level framework. Multi-level oil body peculiar to QB can be divided into four viscosity levels. According to tested real temperature and normal running, proposed viscosity level is selected. For car body engine in the PCV framework, lubricant at the level of QD can be used. For car body installed with circulating emission, selected category of engine oil can be set as L-EQE.

\section{Clearly discriminate causes for degradation}

(I) Accumulation of sundries

Waste falling during combustion, emitted waste gas and remained fuel under the incompletion state will accumulate in the inherent crankcase. Diesel with sulfur element and gasoline with lead accumulate lots of greasy filth and acid substances in normal consumption. Under such circumstances, lubricant highlights clear deterioration inclination.

Accumulated dust, metal debris falling due to abrasion and carbon deposition accumulated in combustion hide a lot of pollution. To change the original oil body quality in most cases, additive under certain specification will be added into 
purchased lubricant. In continuous running, this additive is gradually consumed, resulting in degradation of oil body.

(II) Slow oxidation of oil body

Upon consumption for a long time, lubricant will highlight distinct oxidation inclination. Under the slow state, this oxidation is served as the origin of oil body degradation. Friction carries much heat energy. Moreover, heat derived from the combustor framework promotes the original temperature rise of oil body. Under the situation of temperature rise, lubricant speeds up the oxidation rate. Usually, temperature rise accumulates to a certain point. The measured oxidation rate will remarkably increase. For piston in cylinder sleeve and system, the highest temperature of lubricant will exceed $250^{\circ}$, highlighting the overall inclination of oxidative deterioration. Even if the tested oil temperature is relatively low, oil body will also gradually deteriorate upon accumulated oxidation for a long time. Therefore, new oil body should be frequently replaced.

The fundamental element related to oxidation of oil body also includes the following levels: diversified metals within the alloy framework during running of engine accessories will gradually age upon catalyzing. Splashed oil body will increase the contact area of air. Such elements will promote oxidation and qualitative change.

\section{Analysis on degradation indexes}

(I) Products from oxidation

Upon high temperature oxidation, lubricant will generate attached oxides. Upon the chain reaction in the free radical framework, stable-state final products will be generated. To be specific, diversified products from oxidation can be divided into the following substances:

Firstly, film within the level of colloid and surface; upon condensation reaction, low-molecular compounds will generate high-molecular condensation substances, namely insoluble colloid under certain specification. Upon high temperature extraction, such collected substances form relatively thin film. Such film will attach to inherent piston.

Secondly, carbon deposit obtained from accumulation; incomplete combustion in the combustor will produce lots of carbon deposit. Lubricant falls till into the combustor. Then, it is polluted by sundries in this range. Such carbon deposit is obtained due to incomplete combustion. 
Thirdly, oxidation in the hydrocarbon framework; upon oxidation, hydrocarbon will become lower molecular weight and form oxygen bearing compound, such as alcohol and ketene. A small amount of substances obtained by oxidation will suffer from continuous oxidation. Other residual substances will attach to the surface of inherent air cylinder, escape from oil body and consume lots of oil body. Acid components will also corrode engine accessories.

(II) Changing indexes

Lubricant will be gradually degraded with the consumption of oil body. At the same time, proposed physicochemical indexes will also replace, such as the increase in the original viscosity of oil body, increase in water body covered in oil body, reduced flashing point, progressive increase of acidity and indissolvable sundries. Indexes for evaluation of lubricant cover viscosity. According to the analysis, oxidative degradation and related polymerization flow result in slow volatilization under the state of light constituent and improve the inherent viscosity. However, high rate shearing and gradual attenuation of oil body will also reduce the degree of thickness. New oil body regularly added also results in variation in proposed indexes.

Corrosive acid evaluation index indicates replacement of acidity. It is closely correlated with normal oxidation of oil body. If tested acidity is relatively high, it indicates the oxidation course under the relatively strong state. Oil body will rapidly degrade. Flashing point indicates the attenuation state of fuel oil. It will gradually reduce with the mixing with added oil body. Moisture added into oil body will reduce inherent effect of additive. These indexes within the matching range will be constantly changing with the accumulated mileages.

\section{Reasonably replace lubricant}

Lubricant consumed by engine will gradually deteriorate and degrade. At this time, retained lubricant should be replaced. Emphasis should be laid onto distinct replacement period. Much oil will be consumed, if the proposed period is early. However, engine will be continuously deteriorated, if the proposed period is relatively late. Moreover, the preset service life will be shortened. Multiple correlated indexes should be identified. The replacement time should be specified through test. Accurate values should be identified for oil body viscosity, tested $\mathrm{pH}$ value and insoluble impurities in a certain period. Oil body should be replaced according to the overall guidelines. 
Under normal circumstances, prepared lubricant should be replaced after the car is driven for $5000 \mathrm{~km}$ under preset road conditions. Consideration indexes of oil replacement can cover inherent vehicle model, real performance of engine, sectional road conditions in normal driving and diversified conditions of road. After the run-in period, package indexes of lubricant should be inspected as well. According to the tested value, the oil replacement period in the basic structure should be specified. If relatively large deviation is found in continuous driving, lubricant should be replaced with great care according to actual conditions. For a large-scale passenger car, the oil temperature is relatively high, and it is fast for usual degradation. To this end, the present oil replacement period should be very short.

\section{VII.Conclusion:}

Lubricant added into engine includes diversified specifications. Distinct selection orientation is of great importance for continuous lubrication and control. By-products from combustion of lubricant conceal pollution. Consuming additive will also accelerate degradation of oil body. We should follow the overall guideline of oil replacement, preset proper oil replacement frequency, and select the optimal sampling approach. When lubricant is rather approximate to proposed oil replacement period, we should distinguish and subdivide lubricating indexes and specify oil replacement time. In usual running of engine, real conditions in the periodic stage should be followed. Moreover, proper correction should be made. For lubricant in different characteristics, hierarchical oil replacement period should be determined and feasible industry indexes should be made clear.

\section{Acknowledgments}

This paper is the college-level topic project result of Baoji Vocational Technology College (project No.: 2013010Z) and the topic result of the Shaanxi Society of Technical and Vocational Education (topic No.: SZJYB2014044).

\section{References:}

[1] Shao Linbo, Analysis on Selection of Automobile Engine Lubricant [J], Journal of Kunming Metallurgy College, 2014 (01);

[2] Shao Li, Degradation and Replacement of Automobile Engine Lubricant [J], Chinese Internal Combustion Engine Engineering, 2013 (02);

[3] Geng Xiangjun, Discussion on Selection of Engine Lubricant [J], Shandong 
Internal Combustion Engine, 2012 (01);

[4] Wang Biling, Experimental Research on Degradation Rules of Automobile Engine Lubricant [J], Lubrication Engineering, 2014 (03);

[5] Yao Haifa, Classification and Selection of Automobile Engine Lubricant [J], World Auto, 2014 (03). 